\begin{tabular}{|l|l|}
\hline GC-9 & 101 \\
\hline
\end{tabular}

\title{
ACCURATE CLOSED-FORM SOLUTION OF PROPORTIONAL NAVIGATION FOR MANEUVERING TARGETS
}

\author{
Wagih Abd El-ghany* And Wahid Kassem*
}

ABSTRACT

We present in this paper a method of accurately solving the pure proportional navigation equations in closed form for maneuvering and nonmaneuvering targets. The method is based on a quasilinearization (QL) procedure. The explicit QL solutions presented here are shown to be a generalization of the classical linear (CL) solutions. This generalization results in an improvement in the estimation of missile lateral acceleration and enlarges the validity of the solutions to a wide range of engagement geometries and maneuver levels than is possible with the CL approach. The method outlined here is capable of generating explicit solutions for all trajectory angles, as well as the time of flight and lateral acceleration.

\section{INTRODUCTION}

Proportional navigation (PN) has been widely used as a guidance law for homing applications in the recent decades. It is very simple to implement on-board and is very efficient in a wide variety of geometrical situations. However, the analysis of $\mathrm{PN}$-based guidance systems has been difficult, because the equations governing motion under the PN law are highly nonlinear even under simplifying assumptions. A substantial volume of literature is available on the performance evalution of PN guidance laws from various points of view. Some qualitative treatment and certain particular solutions (CL) have been reported in [1-5], but such treatment has a very little practical value.

The severe restrictions on the engagement geometry and the target maneuver levels, necessary to ensure the validity of classical linear solutions, have been to a large extent obviated by adopting a quasilinear approach [6]. Although such an approach is normally used for numerical iteration, by confining attention only to the terms up to the first derivative and considering only one iteration, it is possible to obtain analytical

* Col. Dr. Eng., Air Defence College.

* Brig. Gen. Prof. Dr., M. T.C. 
expressions for all the trajectory parameters for both nonmaneuvering as well as maneuvering targets. Such a solution has the merit of providing accurate analytical estimates of the trajectory parameters for engagement geometries that are significantly far from the tail-chase and for high target maneuver levels. For these reasons, we'll demonstrate in this paper, the methods used in $[7,8]$ which use a robust linearization procedure to obtain the engagement results that will be useful for this kind of study.

\section{FN EOUATIONS FOR MANEUVERING TARGETS}

We consider the geometry of Fig. 1. The target is assumed to have a constant forward velocity $\mathrm{V}_{\mathbf{T}}$ and a constant lateral acceleration $\mathrm{A}_{\mathbf{T}}$. The differential equations describing the pursuer motion may be written as

$$
\begin{aligned}
& \dot{R}=v_{T} \cos (\theta-k t)-V_{M} \cos (\theta-\phi) \\
& \dot{R} \dot{\theta}=-v_{T} \sin \left(\theta-k_{2} t\right)+v_{M} \sin (\theta-\phi)
\end{aligned}
$$

wher $\mathrm{k}=\mathrm{a}_{\mathrm{T}} / \mathrm{v}_{\mathrm{T}}$ represents the normal turn rate of the target. With $\mathrm{N}$ is the navigation constant, using the definition $\dot{\phi}=\mathrm{N} \dot{\theta}$ of PFN in (1) and (2)

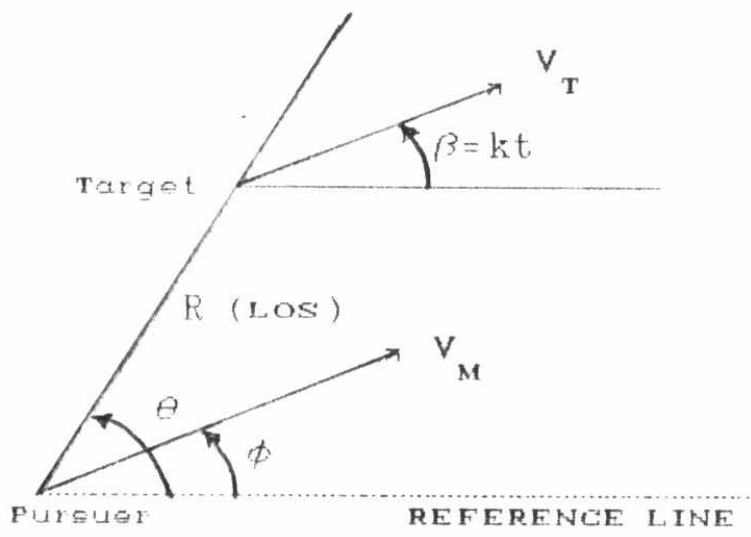

Fig. 1. Geometry of PN for maneuvering target.

$$
\begin{aligned}
& \dot{\mathrm{R}}=\mathrm{v}_{\mathbf{T}} \cos (\theta-\mathrm{kt})-\mathrm{v}_{\mathrm{M}} \cos (\mathrm{b} \theta-\mathrm{c}) \\
& \mathrm{R} \dot{\theta}=-\mathrm{v}_{\mathbf{T}} \sin (\theta-\mathrm{k} t)+\mathrm{v}_{\mathbf{M}} \sin (\mathrm{b} \theta-c)
\end{aligned}
$$

where

$$
b=1-N \text { and } c=\phi_{i}-N \theta_{i}
$$


For the normal range of $\mathrm{PN}$ parameters, the range-to-go $\mathrm{R}$ is a monotonic function of time $t$. Under the assumption of monotonicity, (3) and (4) can be rewritten with $R$ as the independent variable:

$$
\begin{aligned}
\mathrm{T}_{\mathrm{q}} \frac{\mathrm{d} \mathrm{t}^{\prime}}{\mathrm{dR}} & =\frac{1}{\mathrm{v}_{\mathbf{T}} \cos \left(\theta-\mathrm{k}^{\prime} \mathrm{t}^{\prime}\right)-\mathrm{v}_{\mathrm{M}} \cos (\mathrm{b} \theta-\mathrm{c})} \\
& \triangleq \mathrm{d}\left(\theta, \mathrm{k}^{\prime}\right) \\
\mathrm{R} \frac{\mathrm{d} \theta}{\mathrm{dR}} & =\frac{-\mathrm{v}_{\mathrm{T}} \sin \left(\theta-\mathrm{k}^{\prime} \mathrm{t}^{\prime}\right)+\mathrm{v}_{\mathrm{M}} \sin (\mathrm{b} \theta-\mathrm{c})}{\mathrm{v}_{\mathrm{T}} \cos \left(\theta-\mathrm{k}^{\prime} \mathrm{t}^{\prime}\right)-\mathrm{v}_{\mathrm{M}} \cos (\mathrm{b} \theta-\mathrm{c})} \\
& \triangleq \mathrm{g}\left(\theta, \mathrm{k}^{\prime}\right)
\end{aligned}
$$



\section{QUASILINEARIZATION OF PN EQUATIONS}

The coupled equations (6) and (7) are highly nonlinear and aren't solvable in closed form. We derive approximate equivalents of these equations using the QL technique. Expanding the right-hand side (RHS) of (7) and (6), respectively, in Taylor series in function space and truncating after the first derivative term,

$$
\begin{aligned}
& \mathrm{R} \frac{\mathrm{d} \theta_{1}}{\mathrm{dR}}=\mathrm{g}_{0}+\mathrm{h}_{0}\left(\theta_{1}-\theta_{0}\right) \\
& \mathrm{h}_{\mathrm{o}} \triangleq \mathrm{h}\left(\theta_{0}\right) \triangleq \partial \mathrm{g} / \partial \theta \mid \theta=\theta_{0} \\
& \mathrm{~g}_{0} \triangleq \mathrm{g}\left(\theta_{0}\right) \\
& \mathrm{T}_{\mathrm{i}} \frac{\mathrm{dt}}{\mathrm{dR}}=\mathrm{d}_{0}+\mathrm{e}_{\mathrm{o}}\left(\theta_{1}-\theta_{0}\right) \\
& \mathrm{e}_{\mathrm{o}} \triangleq \mathrm{e}\left(\theta_{0}\right) \triangleq \partial \mathrm{d} /\left.\left.\partial \theta\right|_{0}\right|_{0} \\
& \mathrm{~d}_{\mathrm{o}} \triangleq \mathrm{d}\left(\theta_{0}\right)
\end{aligned}
$$


The subscript 0 denotes the initial approximation to the solution and 1 denotes the solution after the first iteration. Although more iterations can be performed to improve accuracy, in this work we stop at the first iteration in order to be able to obtain a closed-form solution. Thus $\theta_{\mathbf{1}}$ is our final solution for the variable $\theta$. To simplify the notation, we replace ${ }_{1}$ with $\theta$ and $t_{1}$ with $t$ in the following treatment.

From definition, and after necessary simplification, $h$ and $\theta$ are obtained as

$$
\begin{aligned}
& h \triangleq \frac{\partial g}{\partial \theta}=\frac{-b v_{M}^{2}-v_{T}^{2}+(1+b) v_{T} v_{M} \cos \left(N \theta+c-k_{i}^{\prime} t^{\prime}\right)}{\left[v_{T} \cos \left(\theta-k^{\prime} t^{\prime}\right)-v_{M} \cos (b \theta-c)\right]^{2}} \\
& +k^{\prime} \frac{d t^{\prime}}{d \theta} \frac{v_{T}^{2}-v_{T} v_{M} \cos \left(N \theta+c-k^{\prime} t^{\prime}\right)}{\left[v_{T} \cos \left(\theta-k_{i}^{\prime} t^{\prime}\right)-v_{M} \cos (b \theta-c]^{2}\right.} \\
& e \triangleq \frac{\partial d}{\partial \theta}=-\frac{-v_{T} \sin \left(\theta-k^{\prime} t^{\prime}\right)+b v_{M} \sin (b \theta-c)}{\left[v_{T} \cos \left(\theta-k^{\prime} t^{\prime}\right)-v_{M} \cos (b \theta-c)\right]^{2}} \\
& +k^{\prime} \frac{d t^{\prime}}{d \theta} \frac{-v_{T} \sin \left(\theta-k^{\prime} t^{\prime}\right)}{\left[v_{T} \cos \left(\theta-k^{\prime} t^{\prime}\right)-v_{M} \cos (b \theta-c)\right]^{2}}
\end{aligned}
$$

Equations (8) and (9) with the definitions in (6), (7), (10), and (11) constitute the approximation to the nonlinear PN equations (6) and (7).

\section{PERTURBATION SOLUTION OF OL EQUATIONS}

The QL equations (8) and (9) are not directly solvable in closed form. A perturbation series method is adopted here to obtain an approximate solution. 'The variables $\theta$ and $t$ ' are expressed as truncated perturbation series:

$$
\begin{aligned}
& \theta\left(R, k^{\prime}\right)=\theta_{N}(R)+k^{\prime} \theta_{M}(R) \\
& t^{\prime}\left(R, k^{\prime}\right)=t_{N}^{\prime}(R)+k^{\prime} t_{M}^{\prime}(R)
\end{aligned}
$$


where subscript $N$ denotes the zeroth-order solution and $M$ denotes the first-order term. It is shown later that the zeroth-order term corresponds to the solution for nonmaneuvering target and the first-order term represents maneuver effects. For the series expansions (12) and (13) to be accurate, $\mathrm{k}^{\prime}$ must be small.

For the QL algorithm, an initial approximation to the solution is necessary. We choose the initial approximations

$$
\begin{aligned}
& \theta_{N O}(R)=\theta_{i} ; \quad \theta_{M O}(R)=0 \\
& t_{N O}^{\prime}=t_{N O} / T_{i}=\frac{\left(R_{i}-R\right) / V_{R_{i L}}}{R_{i} / V_{R_{i L}}}=\frac{R_{i}-R}{R_{i}}
\end{aligned}
$$

Substituting (12) and (13) in (8) and (9) and using (14) in (10) and (11), and after simplification, retaining only the significant terms, we get, on equating the coefficients of $\mathrm{k}^{\prime}$

$$
\begin{aligned}
& R \frac{d \theta_{N}}{d R}=G_{N O}+H_{N O}\left(\theta_{N}-\theta_{i}\right) \\
& R \frac{d \theta_{M}}{d R}=G_{M O}+H_{N O} \theta_{M}+H_{M O}\left(\theta_{N}-\theta_{i}\right) \\
& T_{i} \frac{d t^{\prime}}{d R}=T_{i} \frac{d t_{N}^{\prime}}{d R}=\frac{d t}{d R}=D_{N O}+E_{N O}\left(\theta_{N}-\theta_{i}\right)
\end{aligned}
$$

where

$$
\begin{aligned}
& G_{N O}=\frac{-v_{T} \sin \theta_{i}+v_{M} \sin \left(b \theta_{i}-c\right)}{V_{T} \cos \theta_{i}-v_{M} \cos \left(b \theta_{i}-c\right)}=-\frac{R_{i} \dot{\theta}_{i}}{V_{R_{L_{L}}}} \\
& G_{M O}=-\frac{V_{T}}{V_{R_{i L}}} t_{N O}^{\prime}\left[\cos \theta_{i}-G_{N O} \sin \theta_{i}\right] \\
& H_{N O}=\frac{-b v_{M}^{2}-v_{T}^{2}+(1+b) v_{T} v_{M} \cos \left(N \theta_{i}+c\right)}{\left[v_{T} \cos \theta_{i}-v_{M} \cos \left(b \theta_{i}-c\right)\right]^{2}} \\
& H_{M O}=\frac{-2 H_{N O} V_{T} t_{N O}^{\prime} \sin \theta_{i}}{V_{T} \cos \theta_{i}-V_{M} \cos \left(b \theta_{i}-c\right)}+
\end{aligned}
$$




$$
\begin{gathered}
\frac{(1+b) v_{T_{M}} v_{N o} \sin \left(N \theta_{i}+c\right)}{\left[v_{T} \cos \theta_{i}-v_{M} \cos \left(b \theta_{i}-c\right)\right]^{2}} \\
I_{N O}=\frac{v_{T} \cos \theta_{i}-v_{M} \cos \left(b \theta_{i}-c\right)}{v_{T}}-\frac{1}{v_{{ }_{R}}} \\
E_{\mathrm{NO}}=-\frac{v_{T} \sin \theta_{i}+b v_{M} \sin \left(b \theta_{i}-c\right)}{\left[v_{T} \cos \theta_{i}-v_{M} \cos \left(b \theta_{i}-c\right)\right]^{2}}
\end{gathered}
$$

The zeroth-order equations (15) and (17) corresponds to the nonmaneuvering case and have been solved in closed form in [7]. The solution is reproduced in the following section.

\section{CLOSED-FOFM SOLUTION FOR NONMANEUYERING CASE}

Equations (15) and (17) are constant-coefficient linear differential equations subject to the initial conditions $\theta_{N}\left(R_{i}\right)=\theta_{i}$ and $t\left(R_{i}\right)=0$. From (15), the solution for $\theta_{\mathrm{N}}$ is obtained as

$$
\theta_{N}=\theta_{i}+\frac{G_{N O}}{H_{N O}}\left\{\left[\frac{R_{1}}{\bar{P}_{i}}\right]^{H_{N O}}-1\right\}
$$

Substituting from (24) in (17)

$$
\begin{aligned}
\mathrm{T}_{i} \mathrm{t}^{\prime} & =t=R_{i}\left\{\left[\frac{\mathrm{R}}{\mathrm{R}_{i}}-1\right]\left[\mathrm{D}_{\mathrm{NO}}-\frac{\mathrm{E}_{\mathrm{NO}} \mathrm{G}_{\mathrm{NO}}}{\mathrm{H}_{\mathrm{NO}}}\right]\right. \\
& \left.+\frac{\mathrm{E}_{\mathrm{NO}} \mathrm{G}_{\mathrm{NO}}}{\left.\mathrm{H}_{\mathrm{NO}}{ }_{\mathrm{NO}} \mathrm{H}_{\mathrm{NO}}+1\right)}\left[\left[\frac{\mathrm{R}}{\mathrm{R}_{i}}\right]^{\mathrm{H}_{\mathrm{NO}}+1}-1\right]\right\}
\end{aligned}
$$

The pursuer lateral acceleration $\mathbf{a}_{\mathbf{M}}$, normal to the pursuer velocity vector, is given by

$$
\begin{aligned}
a_{M} & \left.=\left(v_{M} / g\right) d \phi / d t \quad \text { (in } g s\right) \\
& =\left(v_{M} / g\right) N d \theta / d t, \quad \text { using the definition } \dot{t}=\mathrm{N} \dot{\theta} \\
& =\left(v_{M} / g\right) N(d \theta / d R) /(d t / d R)
\end{aligned}
$$


Substituting the values of $d{ }_{\mathrm{N}} / \mathrm{dR}$ and $\mathrm{dt} / \mathrm{dR}$, obtained by differentiation of (24) and (25), for $d \theta / d R$ and $d t / d R$, respectively, in (26),

$$
a_{M}=\frac{N V_{M}}{g_{i}}\left[\frac{G_{N O}\left[\frac{R}{R_{i}}\right]^{H_{N O}^{-1}}}{D_{N O}+\frac{E_{N O} G_{N O}}{H_{N O}}\left[\left[\frac{R}{R_{i}}\right]^{H_{N O}}-1\right]}\right]
$$

Using the last results of (18) and (22),

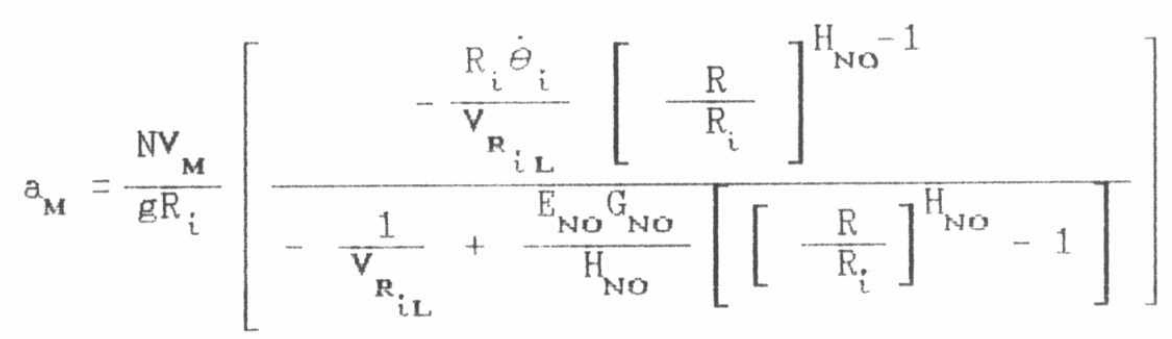

From (17) and (24)

$$
\begin{aligned}
& \frac{d t}{d R}-D_{N O}=E_{N O}\left(\theta_{N}-\theta_{i}\right] ; \quad\left(\theta_{N}-\theta_{i}\right)=\frac{G_{N O}}{H_{N O}}\left[\left[\frac{R}{R_{i}}\right]^{H_{N O}}-1\right] \\
& \frac{E_{N O} G_{N O}}{H_{N O}}\left[\left[\frac{R}{R_{i}}\right]^{H_{N O}}-1\right]=\frac{d t}{d R}-D_{N O}=-\frac{1}{V_{R L}}+\frac{1}{V_{R_{i L}}}
\end{aligned}
$$

The second equality in (29) uses (22) and the definition of $\mathrm{V}_{\mathbf{R L}}$ (where $\mathrm{V}_{\mathbf{R L}}=$ $-\dot{R}$ is the relative closing velocity along LOS ). Using (29) in (28)

$$
a_{M}=\frac{N V_{M} V_{R L}}{g R_{i}} \frac{R_{i} \dot{\theta}_{i}}{V_{R_{i L}}}\left[\frac{R}{R_{i}}\right]^{H_{N O}-1}
$$

Generalized Linear Solution for Lateral Acceleration

The QL derived expression (30) for lateral acceleration $\mathrm{a}_{\mathbf{M}}$ can be cast in a form explicity dependent on the heading error $\Delta \phi_{i}$ relative to the collision course based on the initial geometry. For this, we use the relation [9] 


$$
\dot{\theta}_{i}=-\frac{v_{M} \cos \phi_{c}}{R_{i}} \Delta \phi_{i}
$$

which is valid for small values of $\theta_{i}$ and $\Delta \phi_{i}$. Then, from (30)

$$
a_{M}=-\frac{v_{M}}{g} \frac{v_{R L}}{R_{i}} N^{\prime}\left[\frac{R}{R_{i}}\right]^{H_{N O}-1} \Delta \phi_{l}
$$

which we call the GL form of $\mathrm{a}_{\mathbf{M}}$. Equation (32) is in a form similar to the CL form [5]

$$
\begin{aligned}
a_{M} & =-\frac{v_{M}}{g t_{f}} N^{\prime}\left[1-\frac{t}{t_{f}}\right]^{N^{\prime}-2} \Delta \phi_{i} \\
& =-\frac{v_{M}}{g} \frac{v_{R_{C}}}{\bar{R}_{i}} N^{\prime}\left[\frac{R}{\bar{R}_{i}}\right]^{N^{\prime}-2} \Delta \phi_{i}
\end{aligned}
$$

The second equality is true, since $t_{f}=R_{i} / V_{R_{C}}$ and $t=\left(R_{i}-R\right) / V_{R \subset}$ (where $V_{\mathbf{R} \subset}$ is the constant relative velocity along LOS and $t_{f}$ is the final value of time at intercept). The fact that (32) is a more generalized form of (33) is apparent from a structural comparison of these two expressions for $\mathbf{a}_{\mathbf{M}}$. The parameter $\mathrm{H}_{\mathrm{NO}}$ in the GL solution (32) is the analog of $\mathrm{N}^{\prime}$ in the CL solution (33) with a fixed difference of unity. This is made clear by applying small-angle approximations as shown below.

When $\theta_{i}$ is small, the heading angle $\phi_{c}$ corresponding to the collision course is also small for normal values of $\mathbf{V}_{\mathbf{M}} \mathbf{N}_{\mathbf{T}}$. Further, for small heading errors $\Delta \phi_{i}, \phi_{i}$ would also be small. Then, from the last expression of $(20), H_{N o}$ can be written in the following form.

$$
H_{N_{0}}=-\frac{v_{R_{i}^{2}}}{v_{R_{i L}^{2}}^{2}}+N^{\prime} \frac{V_{R_{i}}}{V_{R_{i L}}}
$$

Since $\mathrm{V}_{\mathrm{R}_{i \mathbf{L}}}$ and $\mathrm{R}_{i} \dot{\theta}_{i}$ are orthogonal, being the components of the relative initial velocity $v_{\mathbf{R}_{i}}$ along and perpendicular to the LOS, we have

$$
v_{R_{i L}}^{2}+R_{i}^{2} \dot{\theta}_{i}^{2}=v_{R_{i}}^{2}
$$


Using the expression (31)

$$
\begin{aligned}
\frac{v_{R_{i}}^{2}}{v_{R_{i L}}^{2}} & =1+\left[\frac{R_{i}}{\dot{R}_{i}}\right]^{2} \frac{v_{M}^{2} \cos ^{2} \phi_{c}}{R_{i}^{2}}\left[\Delta \phi_{i}\right]^{2} \\
& \simeq 1 \text { (neglecting second-order terms in } \Delta \phi_{i} \text { ). }
\end{aligned}
$$

Then, using (36b) in (34)

$$
\mathrm{H}_{\text {No }}=\mathrm{N}^{\prime}-1 \text {. }
$$

This proves that the quantity $\mathrm{H}_{\mathrm{NO}}{ }^{+1}$ of the GL formulation is a generalized replacement for $\mathrm{N}^{\prime}$ in the CL formulation.

Indeed, the generalization involved in GL over CL is a powerful one, in the sence of accommodating a much wider range of pursuit geometries. The linearization assumptions are only weakly used for the GL development, essentially through the LOS rate. In particular, CL requires that the constant-bearing angle $\phi_{c}$ itself be small, in addition to the launch geometry being close to the constant-bearing. In contrast, GL only requires relative closeness between the launch geometry and a constant-bearing geometry, without much restriction on $\phi_{c}$ itself. This fact is also borne out by numerical results which form the next stage of comparison between the two linearized formulations.

The lateral acceleration $a_{M}$ obtained from the GL expression (32) are plotted in Fig. 2. As a reference, for determining the absolute accuracy of the GL results, Fig. 2 also shows the value of $a_{M}$ obtained by an accurate numerical solution of the original nonlinear equations (1) and (2) for $\dot{\theta}$ using a 4/5th-order Runge-Kutta algorithm [10] and substituting this $\dot{\theta}$ in (26) to obtain $a_{M}$ under assumption $\beta=0$. This numerical solution is referred to as the "exact" estimates of $a_{M}$. To put the GL results in comparison with those obtained from the CL formula (33), estimates of $\mathrm{a}_{\mathbf{M}}$ derived from CL are also included in Fig. 2.

To be able to visualize the potential benefits of the generalized linear solution in clearer focus, two specific cases are provided. In both cases we consider an air-to-air homing guided missile situation with a target speed of $300 \mathrm{~m} / \mathrm{s}$, a pursuer speed of $900 \mathrm{~m} / \mathrm{s}$, an initial pursuer-target separation of $5000 \mathrm{~m}$, and two values $\left(10^{\circ}\right.$ and $\left.60^{\circ}\right)$ are considered for the launch angle $\theta_{i}$; the first is chosen to represent the near-tail-chase condition and the second represents a large departure from it. The heading $\Delta \phi_{i}$ error is kept constant at $1.5^{\circ}$. 
In Fig. 2, the commonly used effective navigation constant $N^{\prime}$ values of 2 , 3 , and 4 are considered. It is apparent from the graphs of Fig. 2 that for shallow geometries. where pursuit remains nearly a tail-chase throughout, the CL results are quite close to the exact solution, and the GL results fall between the two. Thus, the GL method produces a marginal improvement in cases where the CL solution is valid. However, for steeper geometries (i.e., larger launch angles), where the CL method is no more valid, the GL method continues to provide useful estimates of the lateral acceleration $\mathbf{a}_{\mathbf{M}}$. 'The results of CL formulation have been included in the last three graphs of Fig. 2 to emphasize this fact, although CL results are not expected to be valid for large launch angles. The GL estimates is especially accurate in the later part of the pursuit, as the normalized time $\mathrm{T} / \mathrm{T}$, goes to one (normalized distance $\mathrm{R} / \mathrm{R}$; goes to zero).

\section{CLOSED-FORM SOLWIION FOR MANEUVERING CASE}

The solution to the first-order equation (16) for $\theta_{\mathbf{M}}$ is obtained as

$$
\begin{aligned}
& \theta_{M}=\frac{V_{T}}{V_{R_{i L}}} \frac{A}{H_{N O}-1}\left[\left[1-\frac{R}{R_{i}}\right]+\frac{1}{H_{N O}}\left[\left[\frac{R}{R_{i}}\right]^{H_{N O}}-1\right]\right] \\
& \text { where } \quad A=\cos \theta_{i}-G_{N O} \sin \theta_{i}, \\
& B=-2 G_{N_{O}} \sin \theta_{i}+\frac{D_{N O} G_{N O}}{H_{N O}} V_{M}(1+b) \sin \left(N \theta_{i}+c\right)
\end{aligned}
$$

Substituting the value of $\theta_{N}$ and $\theta_{M}$ from (24) and (38) in (12), we get

$$
\begin{aligned}
\theta & =\theta_{i}+\frac{G_{N O}}{H_{N O}}\left[\left[\frac{R}{R_{i}}\right]^{H_{N}}-1\right]+\frac{R_{i}}{V_{R_{i L}}^{2}} a_{T} \frac{(A-B)}{H_{N O}-1} \\
& \left.\times\left[1-\frac{R}{R_{i}}\right]+\frac{1}{H_{N O}}\left[\left[\frac{R}{R_{i}}\right]^{H_{N}}-1\right]\right]
\end{aligned}
$$

Differentiating (40) and substituting for $G_{\text {No }}$ from (18) 


$$
\left.\frac{d \theta}{d R}=-\frac{\dot{\theta}_{i}}{V_{R_{i L}}}\left[\frac{R}{R_{i}}\right]^{H_{N O}-1} \frac{a_{r}}{V_{R_{i L}}^{2}} \frac{(A-B)}{H_{N O}-1}\left[\frac{R}{R_{i}}\right]^{H_{N O}-1}-1\right]
$$

and, similarly, differentiating (25)

$$
\frac{d t}{d R}=D_{N O}+\frac{E_{N O} G_{N O}}{H_{N O}}\left[\left[\frac{R}{R_{i}}\right]^{H_{N O}}-1\right]=-\frac{1}{V_{R L}}
$$

When there is no initial heading error, i.e., $\dot{\theta}_{i}=0\left(\Delta \phi_{i}=0\right),(41)$ and (42) reduce, respectively, to

$$
\begin{aligned}
& \frac{\mathrm{d} \theta}{\mathrm{dR}}=\frac{\partial_{\mathrm{T}}}{\mathrm{V}_{\mathrm{R}_{i L}}^{2}} \frac{\cos \theta_{\mathrm{i}}}{\mathrm{H}_{\mathrm{N}}{ }^{-1}}\left[\left[\frac{\mathrm{R}}{\mathrm{R}_{\mathrm{i}}}\right]^{\mathrm{H}_{\mathrm{NO}}{ }^{-1}-1}\right] \\
& \frac{\mathrm{dt}}{\mathrm{dR}}=\mathrm{D}_{\mathrm{N}_{O}}=-\frac{1}{\mathrm{~V}_{\mathrm{R}_{\mathrm{iL}}}}
\end{aligned}
$$

Substituting (41) and (42) in (26), we obtain

$$
\begin{gathered}
a_{M}=N V_{M} \dot{\theta}_{i} \frac{V_{R L}}{V_{R_{i L}}}\left[\frac{R}{R_{l}}\right]^{H_{N} o^{-1}} \\
+\frac{N V_{M} a_{T} V_{R L}}{V_{R_{i L}}^{2}} \frac{A-B}{H_{N o}-1}\left[1-\left[\frac{R}{R_{l}}\right]^{H_{N}{ }^{-1}}\right]
\end{gathered}
$$

In the absence of initial heading error $\left(\Delta \phi_{i}=0\right)$, then $\dot{\theta}_{i}=0$, and (45) reduces to

$$
\mathrm{a}_{\mathrm{M}}=\frac{\mathrm{NV_{M }} \mathrm{a}_{\mathbf{T}}}{\mathrm{V}_{\mathrm{R}}{ }_{\text {iL }}} \frac{\cos \theta_{i}}{\mathrm{H}_{\mathrm{N} O}-1}\left[1-\left[\frac{\mathrm{R}}{\mathrm{R}_{\mathrm{t}}}\right]^{\left.\mathrm{H}_{\mathrm{N}}{ }^{-1}\right]}\right.
$$

Equation (45) is the final general QL solution for the pursuer lateral acceleration $\mathrm{a}_{\mathbf{M}}$ against a target maneuvering with a lateral acceleration $\mathrm{a}_{\mathbf{T}}$. 
In this section we show that the solution represented by (45) and (46) for a maneuvering target is a true generalization of the solutions available for nonmaneuvering case. It is easy to see from (45) that $a_{T}=0$ (i.e., no maneuver) results in the second term vanishing, leaving only the first term which is the nonmaneuvering solution in (30).

\section{Comparison with Linear Solution}

To show the consistency of the general solution (45) with the CL, formulations, we first obtain a compatible form of CL solution. Starting from (1) and (2) and using small-angle approximations on $\Delta \phi, \Delta \beta$, and $\theta$, we obtain [9]

$$
\ddot{\theta}\left(t_{\mathrm{f}}-t\right)-2 \dot{\theta}=-\frac{\mathrm{V}_{\mathrm{M}}}{\mathrm{V}_{\mathrm{R}_{i \mathbf{L}}} \cos \phi} \dot{\phi}+\frac{\mathrm{V}_{\mathrm{T}}}{\mathrm{V}_{\mathrm{R}_{\mathrm{iL}}}} \cos \beta_{\mathrm{i}} \dot{\beta}
$$

where $\beta=\beta_{i}+\Delta \beta$ is equivalent to $k$ tin (1) and ( 2 ) and $\phi=\phi_{c}+\Delta \phi$. The solution of (47) is

$$
\dot{\theta}=-\frac{V_{T} \dot{\beta} \cos \beta_{i}}{V_{R}\left(2-N^{\prime}\right)}\left[1-\left[\frac{t_{f}-t_{f}}{t_{f}}\right]^{N^{\prime}-2}\right]+\dot{\theta}_{i}\left[\frac{t_{f}-t_{f}}{t_{f}}\right]^{N^{\prime}-2}
$$

where $\quad \mathrm{N}^{\prime}=\mathrm{N}\left(\mathrm{V}_{\mathrm{M}} / \mathrm{V}_{\mathrm{R}_{\mathrm{iL}}}\right] \cos \phi_{\mathrm{C}}$ is the effective navigation constant and for small angles,

$$
\dot{\theta}_{i}=-\left[V_{M} \cos \phi_{C} / R_{i}\right] \Delta \phi_{i}
$$

using (26)

$$
\begin{aligned}
& a_{M}=-\frac{V_{M^{M}}}{t_{f}} N^{\prime}\left[\frac{t_{f}-t}{t}\right]^{N^{\prime}-2} \Delta t_{i}+\frac{\cos \beta_{i}}{\cos \phi_{c}} \frac{N^{\prime}}{N^{\prime}-2}\left[1-\left[\frac{t_{f}-t_{t}}{t_{f}}\right]^{N^{\prime}-2}\right] a_{T} \\
& =-\frac{V_{M} V_{R_{i L}}}{R_{i}} N^{\prime}\left[\frac{R}{R_{i}}\right]^{N^{\prime}-2} \Delta \phi_{i}+\frac{N^{\prime}}{N^{\prime}-2} \frac{1}{\cos \phi_{c}}\left[1-\left[\frac{R}{R_{i}}\right]^{N^{\prime}-2}\right] a_{T}
\end{aligned}
$$

where $\beta_{i}$ has been assumed zero without loss of generality and $\phi_{c}$ defines the constant bearing course, or the rectilinear collision course which would be 
necessary for the missile to intercept the target without further maneuver if the velocity following thrust cut-off were to remain constant at $\mathrm{V}_{\mathbf{M}}$. To compare the QL solution with the CL solution, we first recast (45) in terms of $\mathrm{N}^{\prime}$ and $\Delta \phi_{i}$, using (49), as

$$
\begin{gathered}
\mathrm{a}_{\mathbf{M}}=-\frac{\mathrm{V}_{\mathbf{M}} \mathrm{V}_{\mathbf{P L}}}{\mathrm{R}_{i}} \mathrm{~N}^{\prime}\left[\frac{\mathrm{R}}{\mathrm{R}_{\mathrm{i}}}\right]^{\mathrm{H}_{\mathrm{N}}{ }^{-1} \Delta \phi_{i}} \\
+\frac{\mathrm{N}^{\prime}}{\mathrm{H}_{\mathrm{N} \odot}-1}-\frac{\mathrm{A}-\mathrm{B}}{\cos \phi_{C}} \frac{\mathrm{V}_{\mathrm{RL}}}{\mathrm{V}_{\mathrm{R}_{\mathbf{L}}}}\left[1-\left[\frac{\mathrm{R}}{\mathrm{R}_{\mathrm{i}}}\right]^{\mathrm{H}_{\mathrm{N}}-1}\right] \mathrm{a}_{\mathbf{T}}
\end{gathered}
$$

The general solution (51) has two distinct parts, the first corresponding to a nonmaneuvering target and the second providing the contribution due to target maneuver. However, unlike the CL solution (50), where the effects of initial heading error and target maneuver are distinct and uncoupled, here the second term in (51) also contains cross-coupling between $\mathrm{a}_{\mathrm{T}}$ and $\Delta \phi_{\mathrm{i}}$. For a near-tail-chase situation, as assumed for the CL solution, $\theta_{i}$ and $\Delta \phi_{i}$ are small and (51) reduces to

$$
\begin{aligned}
\mathrm{a}_{M} \cong & -\frac{\mathrm{V}_{M} \mathrm{~V}_{\mathrm{R}_{\mathrm{L}}}}{\mathrm{R}_{i}} \mathrm{~N}^{\prime}\left[\frac{\mathrm{R}}{\mathrm{R}_{l}}\right]^{\mathrm{H}_{\mathrm{N}}{ }^{-1} \Delta \phi_{i}} \\
& +\frac{\mathrm{N}^{\prime}}{\mathrm{H}_{\mathrm{N} \circ}{ }^{-1}} \frac{1}{\cos \phi_{C}}\left[1-\left[\frac{\mathrm{R}}{\mathrm{R}_{i}}\right]^{\left.\mathrm{H}^{\mathrm{N}}{ }^{-1}\right] \mathrm{a}_{\mathrm{T}}}\right.
\end{aligned}
$$

It is readily seen that the coupling between $\mathrm{a}_{\mathbf{T}}$ and $\Delta \phi_{i}$ has disappeared in (52) because of the small-angle approximation and the QL result (52) is identical to the $\mathrm{CL}$ result $(50)$ if $\mathrm{H}_{\mathrm{No}}$ in the former is replaced by $\mathrm{N}^{\prime}-1$ in the latter. As has been established, $\mathrm{H}_{\mathrm{NO}}$ is a refinement of the classical effective navigation constant $\mathrm{N}^{\prime}$ and the QL solution for a maneuvering target is a consistent generalization of the CL treatment. 
Simulation Results

Fig. 3 show plots of the lateral acceleration $a_{\mathbf{M}}$ of the pursuer engaging a maneuvering target. The QL estimate of $a_{\mathbf{M}}$ obtained from (45) and (46) for pursuit with and without initial heading error respectively, the CL formulation (50) and the "exact" estimates of a were simulated on a digital computer for comparison purposes. Simulations were made for engagement scenario presented earlier.

In Fig. 3, a most commonly used effective navigation constant $\mathrm{N}^{\prime}$ value of 3 is considered. Fig. 3(a-b) depicts results for small angles of the geometry, representing a near-tail-chase pursuit, and also relatively low target maneuver. For this case, all the three estimates (QL, linear, and "exact") of $\mathrm{a}_{\mathbf{M}}$ are found to be close. However, even for this situation, the QL result shows a much closer coincidence with the exact results than the linear treatment.

In Figs. 3(c-d) and (e-f), the angles and the target maneuver levels are progressively increased to high values. This represents a highly generalized pursuit scenario, with high target maneuvers and engagement geometries that are far from tail-chase and collision-course situations. As seen from Figs. $3(c-f)$, the linear solutions are not valid under such conditions, but the QL technique continues to yield much more accurate results.

\section{CONCLUDTNG REMAPKS}

An attempt has been made to solve the difficult problem of obtaining the trajectory parameters of a projectile pursuing a maneuvering and nonmaneuvering target under the pure proportional guidance strategy.

The validity of the formulation, is also demonstrated from the actual results derived from the formulation, using the exact numerical solution of the original PN equations as the standard. Even under conditions where the linear solutions are also valid, the generalized quasilinear solution of this paper shows a distinct improvement in lateral acceleration estimates; when the conditions become more severe and the linear solution ceases to be applicable, the quasilinear solution still continues to follow the true solution quite faithfully.

The foregoing results demonstrate that, the solution presented in this paper constitutes a definite contribution to the understanding of a very widely used guidance system such as proportional navigation. Furthermore, the approach utilized here for maneuvering and nonmaneuvering targets, offers the potential for analytically handling problems of increasing complexity in the analysis of guidance and navigation systems. 


\section{REFERENCES}

[1] Locke, A. S., "Guidance", Princeton, NJ: Van Nostrand, 1960.

[2] Guelman, M., "A Chalitative Study of Proportional Navigation", IEEE Transactions on Aerospace and Electronic Systems, AES-7, 4 (July 1976), pp 637-643.

[3] Guelman, M., "Proportional Navigation with a Maneuvering Target", IEEE Transactions on Aerospace and Electronic Systems, AES-8, 3 (May 1972), pp 364-371.

[4] Guelman, M., "Missile Acceleration in Proportional Navigation", IEEE Transactions on Aerospace and Electronic Systems, AES-9, 3 (July 1973), PP 462-463.

[5] Jerger,J.J., "Systems Preliminary Design", Princeton, NJ: Van Nostrand, (1960).

[6] Bellman, R., and Kaloba, R., "Quasilinearization and Nonlinear Boundary Value Problems”, New York: American ELsevier, 1965.

[7] Shukla, U. S., and Mahapatra, P. R., "Generalized Linear Solution of Proportional Navigation", IEEE Transactions on Aerospace and Electronic Systems, AES-24, 3 (May 1988), PP 231-238.

[8] Mahapatra, P. R., and Shukla, U. S., "Accurate Solution of Proportional Navigation for Maneuvering Targets", IEEE Transactions on Aerospace and Electronic Systems, AES-25, 1 (Jan. 1989), PP 81-89.

[9] Shinar, J., "Homing of a Rolling Missile Against a Maneuvering - Target", Israel Journal of Technology, II, 3 (1973), PP 117-130.

[10] C. Alkis, "Applied Numerical Methods with Personal Computers", McGraw-Hill Book Co., New York, 1987. 
\begin{tabular}{l|l|}
\hline GC-9 & 117 \\
\hline
\end{tabular}
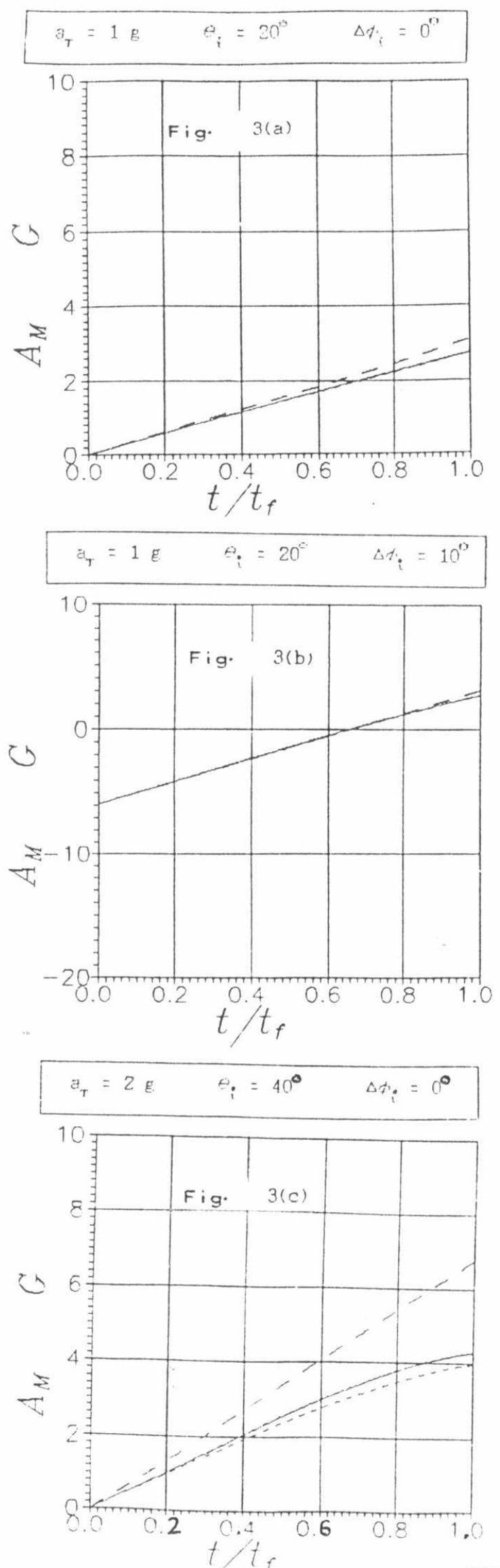

SIXTH ASAT CONFERENCE

2-4 May 1995, CAIRO
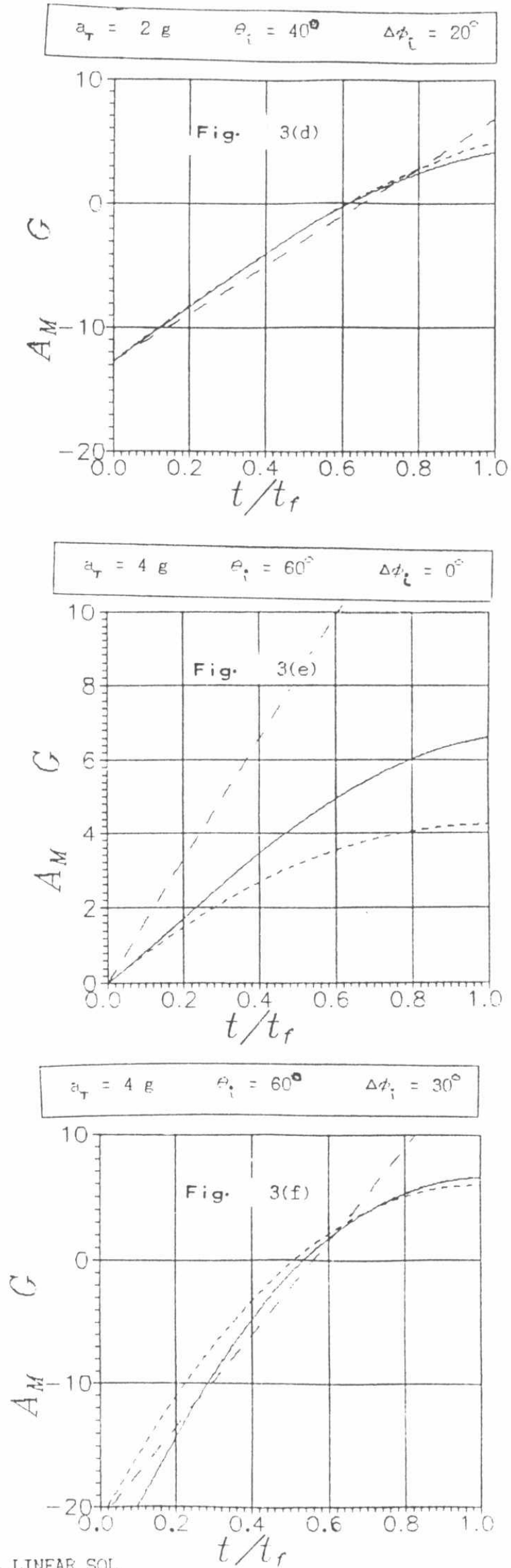

QUASILIMEAR SOL.

FUNGE-YUTTA NUMEFIC SOL 